\title{
Author Index Vol. 27, 1997
}

(B) $=$ Book Review

Abbott, R. 319 Akalan, N. 214 Alarifi, A. 105 Albright, A.L. 40,208 Anthony, D.S. 121 Arginteanu, M. 319 Aronyk, K.E. 166(B) Asai, J. 45 Assaker, R. 211 Atsumi, H. 312 Au, K-S. 304 Aureli, S. 223

Barnes,P.D. 121 Barry, MJ. 40 Bartkowski, H. 71 Baumgartner, J.E. 260

304 Bayer, L.A. 230 Berman, J. 203 Bissonette, D.J. 238 Black, P.M. 312 Boop, F.A. 223 Bourgeois, P. 211 Boyer, R.S. 100 Brockmeyer, D.L. 100

218 Brookshire, B.L. 260,

304 Bruner, J.P. 190 Buxton, N. 108

Carey, CM. 100 Casey, A.T.H. 63 Chabrerie, A. 312 Cherny, W.B. 100 Christenson, J.C. 218 Clifton, G.L. 260 Cochrane, D.D. 56(B) Cohen, A.R. 296 Cohen, L.E. 121 Colak, A. 208 Connors, S. 182 Constantini, S. 153 Coubes, P.A. 268 Crone, K.R. 292

Daly,J.A. 218 Dean, P. 194

Debeugny, S. 211 Del Bigio, M.R. 105 DeLeon, G. 160 Dhellemmes, P. 211 DiRocco, C, 92 Drewek, MJ. 190 Duchowny, M. 194 Duffy, F. 312

Echenne, B.B. 268 Epstein, F.J. 12,34, 153,272

Falkner, L.D. 100 Filston, H.C. 242 Finlay, J.L. 230 Flannery, A.M. 278(B) Folkman, J. 182 Freed, D. 12 Frempong, A. 319 Frim, D.M. 149 Fujimoto, T. 45 Fujimoto, Y. 325 Fulmer, B.B. 242

Gajjar, A. 84 George,T.M. 112(B) Gerszten, P.C. 40 Ginsberg, J. 230 Goh, K.Y.C. 34 Goldman, St. 230 Gonzalez-Gomez, I. 1 Goumnerova, L.C. 149 Grimson, E. 312 Gruber, D.P. 292

Hagen, R. 78 Hariharan, S. 203 Harkness, W.F. 63 Harris, R. 230 Harvey, A.S. 194 Hayward, R.D. 63 Heideman, R.L. 84 Helmers, S. 312 Higbee, R. 176 Higuchi, M. 325 Hladky, J.P. 211 Hokaku, H. 45 Holmes, G. 312

Hoving, E.W. 246 Humbertclaude, V.T.

268 Hunter, J. 50

Iannelli, A. 92 Ikeda, H. 325 İrwin, N. 113

Jackson, P.S. 279,286 Jannetta, P.J. 238 Jayakar, P. 194 Jenkins, E. 218 Johnson, J.H., Jr. 203 Jolesz, F. 312

Kaplan, S.S. 19 Kato, A. 325 Katz, B. 160 Kaufman, B.A. 19,257 Keeve, E. 312 Kellogg,J.X. 28 Khan, S. 230 Kikinis, R. 312 Kim, R. 12 Kim, S.H. 71 Kimmings, E.J. 63 Kleinlugtebeld, A.D. 63 Knepper, P.A. 176 Korgenski, E.K 218 Kosnik, E. 71 Kramer, E.D. 230 Krieger, M.D. 1 Kulkarni, S. 304 Kun, L.E. 84

Lasner, T. 50 Leventon, M.E. 312 Levy, E.I. 238 Levy, M.L. 1,137 Lovely, T. 238

McComb, J.G. 1,137 McLone, D.G. 49,160,

167,176,222,276 Madden, C. 71 Madsen, J.R. 113,121,

$168,279,286$ Magge, S. 168

Magram, G. 277 Malek, A.M. 182 Mapstone, T B. 57 Maugans, T.A. 137 Medlock, M.D. 121

129 Miller, D.C. 153 Miyo, T. 45 Molloy, P. 203 Morrison, G. 194 Muszynski, Ch.A. 272

Nagashima, G. 45 Nakajima, S. 312 Nambiar, U. 272 Noetzel, M.J. 19 Northrup, H. 304

Oakes, W.J. 242 Oruçkaptan, H.H. 214 Ozlen, F. 312

Packer, R.J. 230 Park, T.S. 19,257 Parker, E.C. 223 Pavia, A.T. 218 Phillips, P.C. 203 Piatt, J.H., Jr. 28 Pierce, J. 218 Pittman, T. 78 Pollack, I.F. 208 Prats, A. 194 Punt, J. 108

Radkowski, M.A. 160 Razzaq, A.A. 296 Resnick, D.K, 238 Rezai, A.R. 12 Rinehart, G.C. 78 Riviello, J., Jr. 312 Robain, O. 268 Robertson, R.L. 182 Rorke, L.B. 50,203 Rousseau, J. 211 Rusin, J. 71

Saldana, E. 78 Sanada, Y. 45 Sanford, R.A. 50, 84

Scott, R.M. 121,129

182 Senmevsim, Ö. 214 Seskin, K. 218 Shaver, E.G. 194 Shen, V. 230 Sherburn, E.W. 19 Smith, A. 304 Sorenson, J.M. 260 Squires, L.A. 153 Sutton, L.N. 50,134,

160,203 Suzuki, R. 45

Takahashi, M. 45 Takei, A. 45 Tamburrini, G. 92 Taylor, W.A.S. 63 Teo, C, 223 Thomas,„A.B. 260 Thompson, St. 230 Tulipan, N. 190

Uchiyama, CM. 100

Velasquez, L. 34 Vermeij-Keers, Ch. 246

Wack, D. 71 Walker, M.L. 100 Wang, K-C 176 Weiner, H.L. 12 Wheless, J.W. 260, 304 Whetsell, W.O. 190 Wilkins, R.H. 242 Willmore, L.J. 260 Won,|D.J. 272 Woo, H.H. 12

Yundt, KD. 257 
KAIIGEII

Fax +41613061234 E-Mail karger@karger.ch www.|karger.|com
1998 S. KargerAG, Basel
329

329 